\title{
Alzheimer's Biomarkers are Correlated with Brain Connectivity in Older Adults Differentially during Resting and Task States
}

\author{
Yang Jiang ${ }^{1,2,3 *}$, Haiqing Huang ${ }^{4}$, Erin Abner ${ }^{2,5}$, Lucas S. Broster ${ }^{1}$, Gregory A. Jicha ${ }^{2,6}$, \\ Frederick A. Schmitt ${ }^{2,6}$, Richard Kryscio ${ }^{2,7}$, Anders Andersen ${ }^{3,8}$, David Powell ${ }^{3,8}$, \\ Linda Van Eldik ${ }^{2,8}$, Brian T. Gold ${ }^{2,3,8}$, Peter T. Nelson ${ }^{2}$, Charles Smith ${ }^{2,3,6}$ and \\ Mingzhou Ding ${ }^{4}$

\begin{abstract}
'Department of Behavioral Science, University of Kentucky College of Medicine, Lexington, KY, USA, ${ }^{2}$ Sanders-Brown Center on Aging, University of Kentucky, Lexington, KY, USA, ${ }^{3}$ The Magnetic Resonance Imaging and Spectroscopy Center, University of Kentucky, Lexington, KY, USA, ${ }^{4} \mathrm{~J}$ Crayton Pruitt Family Department of Biomedical Engineering, University of Florida, Gainesville, FL, USA, ${ }^{5}$ Department of Epidemiology, University of Kentucky College of Public Health, Lexington, KY, USA, ${ }^{6}$ Department of Neurology, University of Kentucky College of Medicine, Lexington, KY, USA, ${ }^{7}$ Department of Biostatistics, University of Kentucky College of Public Health, Lexington, KY, USA, ${ }^{8}$ Department of Anatomy and
\end{abstract} \\ Neurobiology, University of Kentucky College of Medicine, Lexington, KY, USA
}

\section{OPEN ACCESS}

Edited by:

P. Hemachandra Reddy,

Texas Tech University, USA

Reviewed by:

Koteswara Rao Valasani,

The University of Kansas, USA

Subodh Kumar,

Texas Tech University Health

Sciences Center, USA

*Correspondence:

Yang Jiang

yjiang@uky.edu

Received: 31 October 2015 Accepted: 19 January 2016 Published: 08 February 2016

Citation:

Jiang Y, Huang $H$, Abner $E$, Broster LS, Jicha GA, Schmitt FA, Kryscio R, Andersen A, Powell D, Van Eldik L, Gold BT, Nelson PT,

Smith $C$ and Ding M (2016)

Alzheimer's Biomarkers are Correlated with Brain Connectivity in Older Adults Differentially during Resting and Task States.

Front. Aging Neurosci. 8:15. doi: 10.3389/fnagi.2016.00015 $\beta$-amyloid $(A \beta)$ plaques and tau-related neurodegeneration are pathologic hallmarks of Alzheimer's disease (AD). The utility of AD biomarkers, including those measured in cerebrospinal fluid (CSF), in predicting future AD risk and cognitive decline is still being refined. Here, we explored potential relationships between functional connectivity (FC) patterns within the default-mode network (DMN), age, CSF biomarkers ( $A \beta_{42}$ and $\left.\mathrm{pTau} \mathrm{T}_{181}\right)$, and cognitive status in older adults. Multiple measures of FC were explored, including a novel time series-based measure [total interdependence (TI)]. In our sample of 27 cognitively normal older adults, no significant associations were found between levels of $A \beta_{42}$ or pTau $_{181}$ and cognitive scores or regional brain volumes. However, we observed several novel relationships between these biomarkers and measures of FC in DMN during both resting-state and a short-term memory task. First, increased connectivity between bilateral anterior middle temporal gyri was associated with higher levels of CSF $A \beta_{42}$ and $\mathrm{A} \beta_{42} / \mathrm{pTau}_{181}$ ratio (reflecting lower AD risk) during both rest and task. Second, increased bilateral parietal connectivity during the short-term memory task, but not during rest, was associated with higher levels of CSF pTau ${ }_{181}$ (reflecting higher AD risk). Third, increased connectivity between left middle temporal and left parietal cortices during the active task was associated with decreased global cognitive status but not CSF biomarkers. Lastly, we found that our new $\mathrm{TI}$ method was more sensitive to the CSF A $\beta_{42}$-connectivity relationship whereas the traditional cross-correlation method was more sensitive to levels of CSF pTau ${ }_{181}$ and cognitive status. With further refinement, resting-state connectivity and task-driven connectivity measures hold promise as non-invasive neuroimaging markers of $A \beta$ and pTau burden in cognitively normal older adults.

Keywords: CSF biomarkers, $\mathbf{A} \beta_{42}$ peptides, $\mathrm{pTau}_{181}$, default-mode network, short-term memory task, global cognitive status, predictors of $A D$ 


\section{INTRODUCTION}

Amyloid plaques and neurofibrillary tangles are the defining pathological features of Alzheimer's disease (AD). The accumulation of $\beta$-amyloid $(A \beta)$ peptide in the brain is considered to indicate preclinical $\mathrm{AD}$ in non-demented individuals (Morris et al., 2006). In animal models, $A \beta$ disrupts neural activity at the synaptic level and at network circuits with other brain regions (Palop and Mucke, 2010). In humans, about $20-50 \%$ of cognitively normal older adults have $A \beta$ deposits imaged with positron emission tomography using Pittsburgh compound B (PIB; Sperling et al., 2009; Quigley et al., 2011).

Although neurofibrillary tangles (tau pathology) and taurelated neurodegeneration are considered the next stage of the $\mathrm{AD}$ progression (Jack et al., 2013), cortical A $\beta$-negative individuals can also have neurodegeneration (as tracked by tau biomarkers; Dickerson and Wolk, 2012; Crary et al., 2014; Kovacs et al., 2016). Furthermore, cognitive functioning in normal older adults is reported to be associated with tau pathology but not with $A \beta$ pathology (Wirth et al., 2013). A recent review on the correlation of AD neuropathological changes with cognitive impairment suggests that the severity of cognitive impairment correlates best with the burden of neocortical neurofibrillary tangles (Nelson et al., 2012). Furthermore, the amount of tau protein in human cerebrospinal fluid (CSF), independently of $A \beta$, is a relatively strong predictor of progression of AD or cognitive decline (Jagust, 2013; Kandimalla et al., 2013; Wirth et al., 2013; Koch et al., 2014).

In cognitively normal older adults, the use of $A \beta$ and tau biomarkers as predictors of functional brain response patterns is still being optimized. The current study investigated the relationship between CSF biomarkers $A \beta_{42}$ or phosphorylated $\mathrm{Tau}_{181}$ (pTau), and functional connectivity $(\mathrm{FC})$ during resting or during a shortterm memory task in cognitively normal older adults. Evidence from functional neuroimaging has revealed decreased brain activity during cognitive tasks, compared to that during resting state, in a group of brain regions that include the posterior precuneus/posterior cingulate cortex (PCC; Brodmann area, BA 31/7), medial prefrontal cortex (mPFC), lateral parietal, and inferior temporal gyrus (BA 20), collectively known as the default-mode network (DMN; Shulman et al., 1997; Raichle et al., 2001). In cognitively normal older adults, stronger deactivations in DMN were found to be associated with better memory performance (Parasuraman and Jiang, 2012). By contrast, AD patients' poor memory performance has been linked to a failure to deactivate the DMN deactivation (Greicius et al., 2004).

Amyloid plaques form within regions of the DMN and the pathology is associated with functional activation differences (Buckner and Vincent, 2007). There are unknown complexities in the relationship between network connectivity during resting state (Biswal et al., 1995) and during task performance, and AD biomarkers (Kandimalla et al., 2011), age, and cognitive status in older persons. Our study investigated whether non-invasive neuroimaging indicators that can predict future $\mathrm{AD}$ risk in cognitively intact older adults. Short-term memory (e.g., working memory) undergoes significant early declines in aging and $\mathrm{AD}$ dementia (Grady et al., 2001). A recent study demonstrated that working memory task-induced deactivation in the DMN can be predicted by resting-state glutamate and GABA concentrations in the PCC/precuneus (Hu et al., 2012). To test the hypothesis that FC among DMN component regions during resting state and task performance are differentially associated with CSF-based levels of $\mathrm{AD}$ biomarkers ( $\mathrm{A} \beta$ and tau) and cognitive status, we analyzed both resting-state and task-driven fMRI data in cognitively normal, well-characterized aged volunteers. For task activation, we developed an older-adult friendly version of a short-term memory task that is sensitive to DMN activity. We also applied a novel time series-based measure of FC called total interdependence (TI) in addition to the traditional cross-correlation (CC) analysis. Based on the literature and our previous work, we predict that resting and task states detect differential default network alterations due to subclinical AD pathology indexed by CSF biomarkers.

\section{MATERIALS AND METHODS}

\section{Participants}

Participants in the current study were healthy, community dwelling, older adults (Mean age 77 years old) enrolled in a longitudinal study of aging and brain health at the University of Kentucky Alzheimer's Disease Center (UK-ADC). These individuals undergo annual neuropsychological testing, clinical assessment, and blood sample collection that are detailed elsewhere (Schmitt et al., 2012). In addition to these procedures, each participant also completes The National Alzheimer's Coordinating Center's Uniform Data Set (Version 2.0; Morris et al., 2006) protocol. Twenty-seven cognitively normal older adults (14 females) participated in the current neuroimaging study. The CSF samples were obtained via lumbar puncture (see below) on the same day that neuroimaging was completed. All research activities were approved by the University of Kentucky Institutional Review Board, and all participants provided written informed consent.

\section{Cognitive and Clinical Assessments}

All participants completed the standard UDS neuropsychological battery (version 1.0) (Weintraub et al., 2009), including the mini-mental state exam (MMSE), within 18 months of the MRI scanning. We computed the UDS T-score, a standardized summary score that measures global cognition, for each participant as described in Mathews et al. (2014). The T-score has a mean of 50 and a SD of 10. A consensus team that included the examining neurologist, neuropsychologist, and psychometrist made the determination of normal cognition based on the results of the UDS assessment.

\section{CSF Collection and Analysis}

Study neurologists collected fasting lumbar CSF samples from participants using a 20 -gage needle and $15-\mathrm{mL}$ sterile polypropylene collection tubes. Samples were stored in single-use $0.5 \mathrm{ml}$ aliquots in a $-80^{\circ} \mathrm{C}$ freezer. The CSF biomarkers $\mathrm{A} \beta_{42}(\mathrm{pg} / \mathrm{ml})$ and $\mathrm{pTau}_{181}(\mathrm{pg} / \mathrm{ml})$ were analyzed in the laboratory of Mary Jo LaDu at the University of Illinois at Chicago using INNOTEST ELISA kits by Innogenetics, Gent, Belgium. Detailed procedures are described in Tai et al. (2013). 


\section{Neuroimaging Procedures}

MRI images were obtained with a 3-T Siemens Trio Scanner at the MRISC of the University of Kentucky using a 32-channel head coil. High-resolution anatomic images (20 min 3D MPRAGE) were acquired using a rapid gradient echo acquisition sequence (acquisition matrix $256 \times 256 \times 176$, isotropic $1 \mathrm{~mm}$ voxels, field of view $256 \mathrm{~mm}$, repetition time $2530 \mathrm{~ms}$, echo time $2.26 \mathrm{~ms}$ ). The resting-state functional MRI (fMRI) was acquired with the following parameters: $\mathrm{TR}=2 \mathrm{~s}$; $\mathrm{TE}=30 \mathrm{~ms}$; flip angle $=76^{\circ} ; 39$ axial slices; FOV $=224 \mathrm{~mm} \times 224 \mathrm{~mm}$; slice thickness $=3.5 \mathrm{~mm}$; matrix $=64 \times 64$; bandwidth $=2056 \mathrm{~Hz} / \mathrm{Px}$. A total of 200 volumes of resting-state data were acquired. The task-based fMRI scan was acquired using a $\mathrm{T} 2{ }^{\star}$-weighted gradient echo EPI sequence $\mathrm{TR}=2 \mathrm{~s} ; \mathrm{E}=30 \mathrm{~ms}$; flip angle $=81^{\circ} ; 39$ axial slices; FOV $=224 \mathrm{~mm} \times 224 \mathrm{~mm}$; slice thickness $=3.5 \mathrm{~mm}$; matrix $=64 \times 64$; bandwidth $=2056 \mathrm{~Hz} / \mathrm{Px}$.

\section{The Short-Term Memory Task}

The participants performed a modified version of a visual working memory task (delayed-match-to-sample task with repeated retrieval of memory targets and distractors) that has been validated in healthy young subjects (Jiang et al., 2000). The task used two-dimensional pictures of common objects taken from Snodgrass and Vanderwart (1980). In the typical delayed matchto-sample paradigm, the subjects were first shown an item to hold in working memory at the beginning of a trial, and then determine whether a later encountered test item is a match or non-match. In the current 10-min older-adult friendly version, two sample pictures were encoded for each given trial. This reduces scanning time for older adults and increases the number of matches with balanced number of non-matches. Task-induced default network activity was calculated as a whole, with multiple cognitive components, in contrast to resting-state connectivity. Details of the event-related task are presented in Supplementary Material. Participants were trained on the memory task before the scanning session.

\section{Functional Connectivity Analysis of Resting State and Task}

Resting-state fMRI and memory task fMRI data were preprocessed for time alignment and scanning artifact removal. For each fMRI run, the first five dummy scans (subjects were reading instructions) were discarded to eliminate typical transients at the beginning of each run. The remaining functional images were processed using SPM8. ${ }^{1}$ Slice timing of each functional scan was corrected to compensate for acquisition delays across slices. Motion artifacts were also estimated and corrected by realigning all functional images to the first image. The motion corrected functional images were co-registered onto the T1 structural image, then normalized to the standard MNI T1 template, and resampled into $3 \mathrm{~mm}$ isotropic voxels. Functional images in the MNI template space were spatially smoothed with an $8 \mathrm{~mm}$ fullwidth at half maximum (FWHM) isotropic Gaussian kernel.
Group independent component analysis (ICA) implemented in the GIFT Toolbox ${ }^{2}$ was applied to the smoothed resting-state fMRI images. The components that contained mPFC, PCC/Precunues, bilateral angular gyrus (AG) in the inferior parietal gyri (BA 39), and bilateral anterior temporal gyri [middle temporal Gyri (MTG), BA21, and BA20], which are known regions of the DMN, were identified and selected. For each DMN region, a region of interest (ROI) was defined to contain voxels within a sphere of $5 \mathrm{~mm}$ in radius centered at the voxel with the local maximum $t$-value.

Time series were extracted from all the voxels in each spherical ROI for both resting-state and task data. Residuals from nine nuisance signals, including six movement variables and three averaged signals from WM, CSF, and whole brain, were produced by regression with the time series. For resting-state data, time series were band pass filtered between 0.01 and $0.1 \mathrm{~Hz}$ with a finite impulse response (FIR) filter. For task functional data, each run was divided into two blocks. A total of four blocks were created from 2 runs of task fMRI. Each block contained four trials. After removing the global effects from the BOLD time series, the temporal mean was removed from each of the four blocks of time series, and the task-evoked effect was also removed by subtracting the ensemble mean of the four blocks of time series. These mean-removed time series were then used for connectivity analysis (Ding et al., 2006).

Two measures of FC, i.e., CC and TI were computed for both resting-state and task data. Although $\mathrm{CC}$ is the most commonly used measure for FC analysis, it only exploits the zero-lag covariance structure of the data. FMRI BOLD signals, however, are time series. A hallmark of a time series is the presence of temporal correlations at non-zero lags (Wen et al., 2012). Thus, in addition to the commonly used CC method, we also applied TI as another FC measure, which takes into account the temporal relationship beyond the zerolag (Wen et al., 2012). (see Supplementary Material for additional description and equations for the TI method).

\section{Statistical Analysis}

We used general linear regression to identify connectivity measures significantly associated with cognition and CSF-based measures of $\mathrm{A} \beta_{42}(\mathrm{pg} / \mathrm{ml})$ and $\mathrm{pTau}{ }_{181}(\mathrm{pg} / \mathrm{ml})$. The UDS T-score was used in the regression models rather than MMSE due to the wider range of scores. Normal distribution of these endpoints was assessed with the Shapiro-Wilk test. We modeled resting and task-based connectivity measures independently. Analyses were further stratified by measure type (CC or TI) such that four models were fit to the data for each outcome (UDS T-score, $\mathrm{A} \beta_{42}$ $[\mathrm{pg} / \mathrm{ml}]$, and $\left.\mathrm{pTau}_{181}[\mathrm{pg} / \mathrm{ml}]\right)$ : resting state, CC connectivity; resting state, TI connectivity; task, CC connectivity; and task, TI connectivity. In each case, the initial model included all CC or TI connectivity measures relevant to resting or task, along with participant age (in years), sex (male $=0$, female $=1$ ), and years of education.

To account for multi-colinearity among the connectivity measures, as well as the high number of predictors versus subjects, we used the least absolute shrinkage and selection operator (LASSO) 
to identify the best subset of candidate predictors for each model (Tibshirani, 1996). The tuning parameter for each LASSO model was selected via cross-validation using PROC GLMSELECT (SAS $9.3^{\circledR}$ ). For instance, to estimate mean $A \beta_{42}$ using CC connectivity measures, the initial regression equation prior to application of the LASSO was as follows: $\mathrm{A} \beta_{42}=\beta_{0}+\beta_{1}{ }^{\star}$ age $+\beta_{2}{ }^{*}$ sex $+\beta_{3}{ }^{*}$ education $+\beta_{4}{ }^{\star} \mathrm{PCC}-\mathrm{mPFC}_{\mathrm{CC}}+\beta_{5}{ }^{*} \mathrm{PCC}-\mathrm{lAG}_{\mathrm{CC}}+\beta_{6}{ }^{\star} \mathrm{PCC}-\mathrm{rAG} \mathrm{CC}+\beta_{7}{ }^{\star} \mathrm{PCC}-$ $1 \mathrm{MTG}_{\mathrm{CC}}+\beta_{8}{ }^{\star}$ PCC $-\mathrm{rMTG} \mathrm{CC}+\beta_{9}{ }^{\star} \mathrm{mPFC}-1 \mathrm{AG} \mathrm{CC}+\beta_{10}{ }^{\star} \mathrm{mPFC}-$ $\mathrm{rAG}_{\mathrm{CC}}+\beta_{11}{ }^{*} \mathrm{mPFC}-\mathrm{lMTG}_{\mathrm{CC}}+\beta_{12}{ }^{*} \mathrm{mPFC}-\mathrm{rMTG} \mathrm{CC}+\beta_{13}{ }^{*} \mathrm{lAG}-$ $\mathrm{rAG}_{\mathrm{CC}}+\beta_{14}{ }^{*} \mathrm{lAG}-\mathrm{lMTG}_{\mathrm{CC}}+\beta_{15}{ }^{*} \mathrm{lAG}-\mathrm{rMTG}_{\mathrm{CC}}+\beta_{16}{ }^{*} \mathrm{rAG}-\mathrm{lMT}-$ $\mathrm{G}_{\mathrm{CC}}+\beta_{17}{ }^{*} \mathrm{rAG}-\mathrm{rMTG} \mathrm{CC}+\beta_{18}{ }^{*} \mathrm{lMTG}-\mathrm{rMTG}$ CC. Backwards selection was then used to fit the final models on the reduced set of predictors using PROC REG (SAS 9.3 ${ }^{\circledR}$ ). LASSO reduced the number of predictors to be placed into backwards regression models from 18 to a median of 4 (range 2-9). We retained variables in the final models if they met statistical significance at 0.05 . No variables were forced into the models.

\section{RESULTS}

\section{Characteristics of the Participants}

All 27 participants were cognitively intact at the time of the neuroimaging and CSF biomarker study. The means and SDs of age (77), education and neuropsychological scores (MMSE, UDS $T$-scores), and their CSF biomarkers ( $\mathrm{A} \beta_{42}, \mathrm{pTau}{ }_{181}$, and $\mathrm{A} \beta_{42} /$ $\mathrm{pTau}_{181}$ ) are reported in Table 1 . The median of the MMSE scores was the ceiling score of 30, with interquartile range of 27-30. Mean UDS $T$-scores were over 0.5 SDs above the mean of 50 (56.8).

Mean $\mathrm{A} \beta_{42}$ was $496 \mathrm{pg} / \mathrm{ml}$ for this sample. The method used for detection of $A \beta_{42}$ level in the CSF sample established values $>500 \mathrm{pg} / \mathrm{ml}$ as $\mathrm{A} \beta$-normal (Sjögren et al., 2001). Using this standard, 12 out of 27 participants were "high-risk" for future AD. Mean pTau ${ }_{181}$ was $65.7 \mathrm{pg} / \mathrm{ml}$.

\section{DMN Regions of Interest}

Based on the ICA results shown in Figure 1, six DMN ROIs were selected in this study, including PCC/Precuneus (BA 23), mPFC (BA10), bilateral AG (BA19\&39), and bilateral middle temporal gyri (BA21). Additional information on the number of voxels in each ROI and statistical significance of activation is also presented in Figure 1.

\begin{tabular}{|c|c|}
\hline Characteristics & Summary \\
\hline Age at fMRI & $77.1 \pm 7.4$ \\
\hline Education & $16.8 \pm 2.3$ \\
\hline Male (\%) & 48.2 \\
\hline MMSE (global cognition) & $29.5 \pm 0.7$ \\
\hline UDS T-score (global cognition) & $56.8 \pm 6.9$ \\
\hline $\mathrm{A} \beta_{42}(\mathrm{pg} / \mathrm{ml})$ & $496.0 \pm 208.7$ \\
\hline $\operatorname{pTau}_{181}(\mathrm{pg} / \mathrm{ml})$ & $65.7 \pm 19.5$ \\
\hline $\mathrm{A} \beta_{42}(\mathrm{pg} / \mathrm{ml}) / \mathrm{pTau}{ }_{181}(\mathrm{pg} / \mathrm{ml})$ & $8.1 \pm 3.6$ \\
\hline
\end{tabular}

All participants were cognitively normal at the time of biomarker collection and cognitive testing.

\section{Accuracy of the Performance during the Short-Term Memory Task}

The accuracy of memory performance was examined to ensure that the participants were actually engaging on cognitive activity during task scanning. The overall accuracy for the task was about $90 \%$. Two of the older participants did the task well but did not switch hands to indicate target match/non-match response between the two 5-min fMRI memory runs. However, for the purpose of the current study, calculated accuracy is less important than participant engagement in the task (short-term memory encoding, retrieval, and visual fixation/perception), allowing contrast with the resting state and calculation of FC over the entire task period.

\section{CSF A $\beta_{42}, A \beta_{42} /$ pTau $_{181}$ Ratio, Regional Brain Volume, DMN Connectivity, and Cognition}

In our sample of 27 cognitively intact older adults, age was not significantly correlated with CSF A $\beta_{42}(r=0.05, p=0.81)$, pTau $\operatorname{Tau}_{181}$ $(r=0.11, p=0.59)$ or UDS T-score $(r=-0.16, p=0.42)$ when FC was assessed using the CC method. However, age was correlated negatively with $\mathrm{A} \beta_{42}$ when FC was assessed by the TI method only during task (Table 3, $p<0.01$ ). We included age in all regression models (Tables 2 and 3 ). Importantly, no significant correlations were found among levels of $\mathrm{A} \beta_{42}, \mathrm{pTau}{ }_{181}$, regional brain volumes, or UDS T-scores. Brain volume analysis and results were reported previously in Gold et al. (2014).

To avoid spurious results due to Type 1 error or inadequate statistical power (Biswal et al., 2010), we set criteria that significant correlations are reported only if they meet one of two criteria: significant results in the statistical model stronger than age-related $\mathrm{A} \beta$ deposits at the $p<0.01$ level (Table 3); or, consistent results replicated in both methods (CC and TI) or during both states (resting state and task). Notably, analyses of both resting and task data revealed that FC between bilateral anterior MTG (MTG-DMN nodes, BA 21) is significantly associated with $\mathrm{A} \beta_{42}$ levels $(p<0.01$; " $\mathrm{b}$ " or " $c$ " in the tables) as well as $\mathrm{A} \beta / \mathrm{pTau}_{181}$ ratio during resting state and task $(\beta=0.42$ in both models, $p<0.05$; complete model results not shown). Taking age into account, CSF A $\beta_{42}$ level was positively correlated with bilateral connectivity of the anterior lateral temporal lobes (IMTG-rMTG) using either CC or TI analysis. Figure 2 shows that six out of eight analyses revealed that lMTG-rMTG is significantly associated with level of $A \beta_{42}$ or $A \beta_{42} / \mathrm{pTau}_{181}$ ratio. In other words, cognitively intact older individuals with increased bilateral MTG connectivity were associated with lower risk of $\beta$-amyloid deposits.

Another positive correlation with $\mathrm{A} \beta_{42}$ was found between DMN FC of left MTG and right AG in parietal lobe (Tables 2 and 3; rAG-lMTG, TI connectivity measures). That is, stronger connectivity indicates increased level of CSF A $\beta_{42}$, implying less deposition of $\beta$-amyloid and, therefore, lower $\mathrm{AD}$ risk.

Brain connectivity indicating higher risk of $\beta$-amyloid deposits was also found. The strongest associations were during task: left middle temporal - mid frontal and left temporal-parietal negatively correlated with $A \beta_{42}$ during the short-term memory task. The mPFC-lMTG connectivity during task (Table 3), and the lAGIMTG connectivity during rest (Table 2), were negatively correlated 


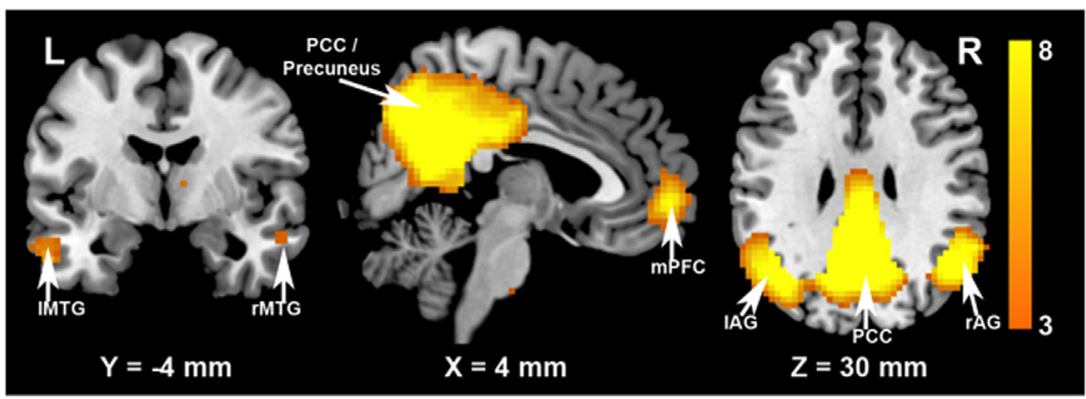

\begin{tabular}{|c|c|c|c|c|c|c|}
\hline \multirow{2}{*}{$\begin{array}{l}\text { Anatomical } \\
\text { Structure }\end{array}$} & \multirow{2}{*}{$\begin{array}{c}\text { Size } \\
\text { (voxels) }\end{array}$} & \multicolumn{3}{|c|}{ MNI Coordinates } & \multirow[t]{2}{*}{ T-value } & \multirow{2}{*}{$\begin{array}{c}\text { Brodmann } \\
\text { Area }\end{array}$} \\
\hline & & $x$ & $\mathrm{Y}$ & $Z$ & & \\
\hline PCC & 3949 & -15 & -60 & 24 & 11.46 & 23 \\
\hline mPFC & 258 & 3 & 57 & -3 & 7.97 & 10 \\
\hline IAG & 432 & -36 & -69 & 36 & 10.28 & $19 / 39$ \\
\hline rAG & 804 & 45 & -66 & 36 & 10.46 & 39 \\
\hline IMTG & 214 & -54 & -3 & -30 & 6.05 & 21 \\
\hline rMTG & 9 & 57 & 0 & -21 & 3.92 & 21 \\
\hline
\end{tabular}

FIGURE 1 | Independent component analysis (ICA) of DMN. Cortical regions of mPFC, PCC/Precuneus, bilateral angular gyrus (AG), and bilateral middle temporal Gyri (MTG) are activated.

TABLE 2 | Standardized beta coefficients for $A \beta_{42}$ models, including resting-state functional connectivity measures [cross-correlation (CC) and total interdependence (TI)].

\begin{tabular}{|c|c|c|}
\hline \multirow[b]{2}{*}{ Model adj. $R^{2}$} & \multicolumn{2}{|c|}{$A \beta_{42}(p g / m l)$} \\
\hline & 0.390 & 0.580 \\
\hline Connectivity measures & $\mathrm{CC}$ & TI \\
\hline \multicolumn{3}{|l|}{ Predictor } \\
\hline \multicolumn{3}{|l|}{ Age, 1 year } \\
\hline \multicolumn{3}{|l|}{ Sex } \\
\hline \multicolumn{3}{|l|}{ Education, 1 year } \\
\hline \multicolumn{3}{|l|}{ PCC-mPFC } \\
\hline \multicolumn{3}{|l|}{ PCC-IAG } \\
\hline \multicolumn{3}{|l|}{ PCC-rAG } \\
\hline \multicolumn{3}{|l|}{ PCC-IMTG } \\
\hline \multicolumn{3}{|l|}{ PCC-rMTG } \\
\hline \multicolumn{3}{|l|}{ mPFC-IAG } \\
\hline mPFC-rAG & $0.379^{a}$ & $0.592^{b}$ \\
\hline \multicolumn{3}{|l|}{ mPFC-IMTG } \\
\hline \multicolumn{3}{|l|}{ mPFC-rMTG } \\
\hline \multicolumn{3}{|l|}{ IAG-rAG } \\
\hline IAG-IMTG & & $-1.00^{b}$ \\
\hline \multicolumn{3}{|l|}{ IAG-rMTG } \\
\hline rAG-IMTG & & $0.656^{a}$ \\
\hline \multicolumn{3}{|l|}{ rAG-rMTG } \\
\hline IMTG-rMTG & $0.474^{b}$ & $0.839^{c}$ \\
\hline
\end{tabular}

The predictors are significant at the following: ${ }^{a} p<0.05$; ${ }^{b} p<0.01 ;{ }^{c} p<0.0001$. Standardized coefficients estimate the number of SDs the dependent variable will change given a one-SD increase in the predictor variable. All predictors included in the initial models are listed in the tables; predictors with no beta coefficients were excluded from the final models. There were no significant associations between resting-state connectivity measures and UDS Tscore or pTau 181
TABLE 3 | Standardized beta coefficients for $A \beta_{42}$ and $p T{ }_{181}$ models, including task-driven fMRI functional connectivity measures [crosscorrelation (CC) and total interdependence (TI)].

\begin{tabular}{|c|c|c|c|c|}
\hline \multirow[b]{3}{*}{ Model Adj. $R^{2}$} & \multicolumn{4}{|c|}{ Dependent variable } \\
\hline & \multicolumn{2}{|c|}{$\mathrm{A} \beta_{42}(\mathrm{pg} / \mathrm{ml})$} & \multicolumn{2}{|c|}{$\mathrm{pTau}_{181}(\mathrm{pg} / \mathrm{ml})$} \\
\hline & 0.317 & 0.646 & 0.359 & 0.149 \\
\hline Connectivity measures & $\mathrm{CC}$ & $\mathrm{TI}$ & $\mathrm{CC}$ & TI \\
\hline \multicolumn{5}{|l|}{ Predictor } \\
\hline Age, 1 year & & $-0.668^{b}$ & & \\
\hline \multicolumn{5}{|l|}{ Sex } \\
\hline \multicolumn{5}{|l|}{ Education, 1 year } \\
\hline PCC-mPFC & & $0.430^{\mathrm{b}}$ & & \\
\hline \multicolumn{5}{|l|}{ PCC-IAG } \\
\hline PCC-rAG & & $-0.526^{b}$ & & \\
\hline \multicolumn{5}{|l|}{ PCC-IMTG } \\
\hline PCC-rMTG & $-0.395^{\mathrm{a}}$ & & & \\
\hline \multicolumn{5}{|l|}{ mPFC-IAG } \\
\hline \multicolumn{5}{|l|}{ mPFC-rAG } \\
\hline mPFC-IMTG & $-0.418^{a}$ & $-0.901^{\mathrm{c}}$ & $-0.401^{a}$ & \\
\hline \multicolumn{5}{|l|}{ mPFC-rMTG } \\
\hline IAG-rAG & & & $0.591^{b}$ & $0.428^{a}$ \\
\hline \multicolumn{5}{|l|}{ IAG-IMTG } \\
\hline \multicolumn{5}{|l|}{ IAG-rMTG } \\
\hline rAG-IMTG & & $1.158^{c}$ & & \\
\hline \multicolumn{5}{|l|}{ rAG-rMTG } \\
\hline IMTG-rMTG & $0.623^{b}$ & & & \\
\hline
\end{tabular}

The predictors are significant at the following: ${ }^{a} p<0.05$ level.; ${ }^{b} p<0.01$ level.; ${ }^{c} p<0.0001$ level.

Standardized coefficients estimate the number of SDs the dependent variable will change given a one-SD increase in the predictor variable. All predictors included in the initial models are listed in the tables; predictors with no beta coefficients were excluded from the final models. 

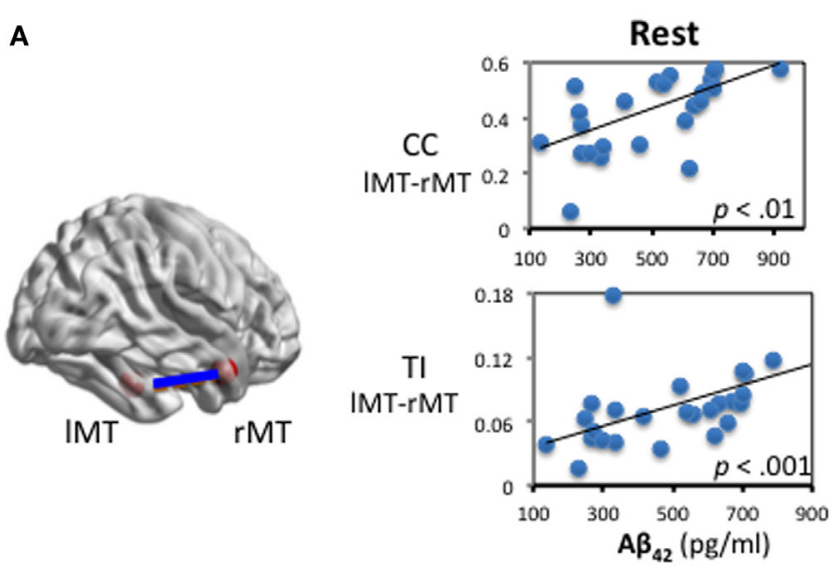

B
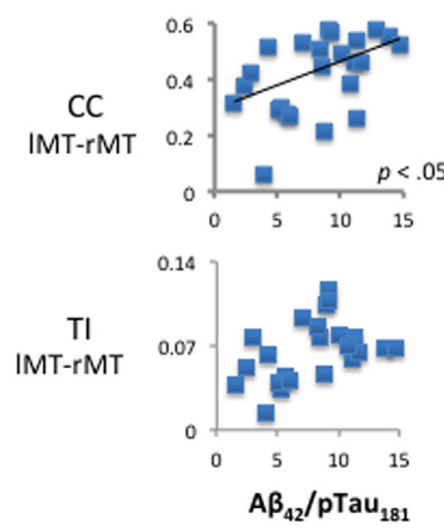

Task
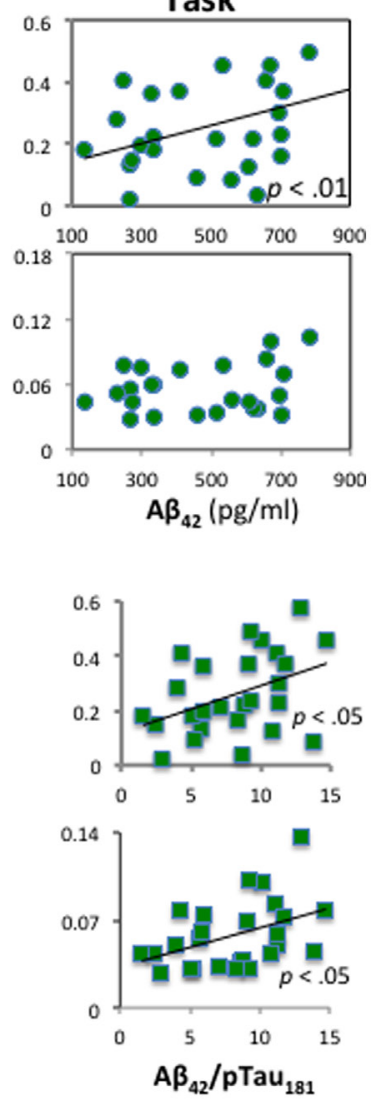

FIGURE 2 | Functional connectivity of bilateral temporal gyri and CSF $\mathbf{A} \beta_{42}$ and $\mathbf{A} \beta_{42} / \mathrm{pTau}_{181}$. (A). $A \beta_{42}$ and bilateral anterior MTG connectivity. (B) $A \beta_{42} /$ pTau ${ }_{181}$ ratio and bilateral anterior MTG connectivity. Cognitively intact older individuals with increased bilateral MTG were associated with low risk of $\beta$-amyloid accumulations.

with $\mathrm{A} \beta_{42 .}$. In other words, stronger connections were associated with lower level of CSF A $\beta_{42}$, i.e., higher $\beta$-amyloid burden.

\section{CSF pTau ${ }_{181}$ Biomarkes and Task-Driven Functional Connectivity}

Cerebrospinal fluid $\mathrm{pTau}_{181}$, but not $\mathrm{A} \beta_{42}$, was strongly linked to DMN FC during the short-term memory task (Table 3, lAG-rAG). Figure $3 \mathrm{~A}$ shows the significant $p$-values ( $y$-axis) of the correlation between $\mathrm{pTau}_{181}$ and connectivity of brain regions only during task. No significant correlations between $\mathrm{pTau}_{181}$ and connectivity measures were observed for the resting-state data (Figure 3A). Figure 3B shows increased $\mathrm{pTau}_{181}$ (higher risk for $\mathrm{AD}$ ) is positively correlated with bilateral AG. Stronger bilateral AG connectivity was associated with higher levels of CSF pTau ${ }_{181}$ $(\mathrm{pg} / \mathrm{ml})$. As shown in Figure 3B, individuals with stronger FC of

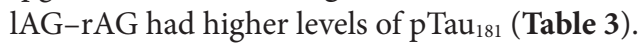

\section{CSF Biomarkers, DMN Connectivity as Predictors for Global Cognition}

We found that global cognition, as measured by the UDS $T$-score, was not correlated with bilateral anterior temporal or bilateral parietal connectivity, nor CSF $\mathrm{A} \beta_{42}$ or pTau ${ }_{181}$ levels. UDS $T$-scores were negatively correlated with IMTG-PCC connectivity $(\beta=-0.557, p<0.01$; complete model results not shown) during the memory task (Figure 4A) but not during rest (Figure 4B). Participants with stronger FC between PCC and left MTG had lower $T$-scores, suggesting a more general role of this connectivity in cognitive status. However, IMTG-PCC connectivity was not associated with CSF $\mathrm{A} \beta_{42}$ or pTau, putative biomarker links with $\mathrm{AD}$ pathology. We further tested bilateral parietal AG (Figure 4C) connectivity correlated with pTau (indicating neural degeneration), but found no correlation to $T$-scores (Figure 4D). There were no significant associations among the remaining DMN connectivity during resting-state and global cognitive function. Given the number of variables and models tested, Type 1 error is possible.

\section{DISCUSSION}

\section{Summary of the Results}

We examined relationships between DMN functional brain connectivity, CSF biomarkers of AD pathology, and global 
A

CSF pTau ${ }_{181} \&$ DMN during Rest \& Task

Rest

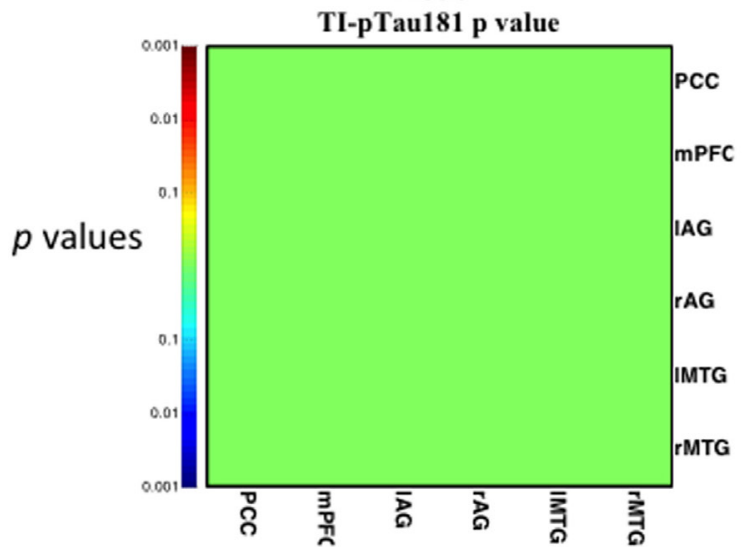

B Bilateral parietal connectivity during task \& pTau $_{181}$
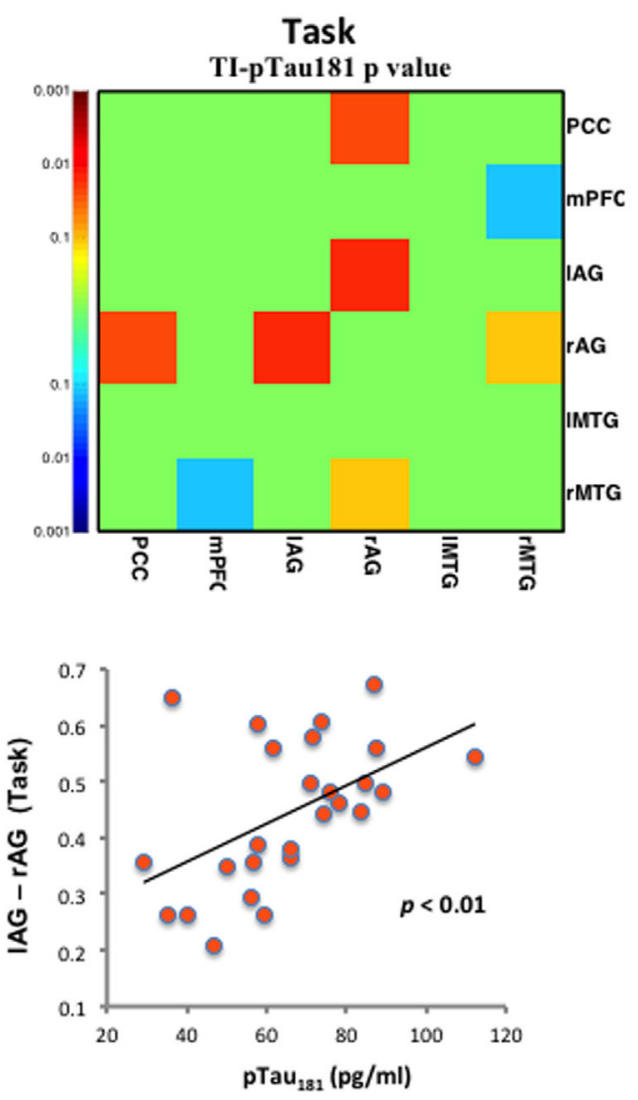

FIGURE 3 | CSF pTau ${ }_{181}$ level and functional connectivity during DMN. (A) CSF pTau ${ }_{181}$ during rest and task at various significant $p$-values; the significant $p$-values ( $y$-axis) of the correlation between pTau ${ }_{181}$ and connectivity of brain regions were found only during task, but not during resting state; (B) pTau ${ }_{181}$ and bilateral inferior parietal connectivity during task. Individuals with stronger IAG-rAG have higher level of pTau ${ }_{181}$, which indicates higher risk of tau-related neural degeneration.

cognition in cognitively intact older adults. The overall results are summarized in Figure 5. We report consistent results from six analyses which indicate that connectivity between bilateral anterior middle temporal lobe (lMTG-rMTG; BA 21) correlates positively with $\mathrm{CSF} A \beta_{42}$ and $\mathrm{A} \beta_{42} / \mathrm{pTau}_{181}$ ratio (Figure 2). This relationship was seen in the resting state and during a memory task. The left middle temporal cortex appears to be a hub of FC related to $\mathrm{A} \beta_{42}$ (Figure 5).

Levels of CSF pTau $_{181}$ showed no association with restingstate connectivity but were correlated positively with bilateral parietal (lAG-rAG) connectivity during a short-term memory task. Additionally, global cognitive status negatively correlated with connectivity between left MTG and PCC only during the memory task, but this connectivity was not associated with AD biomarkers CSF $A \beta_{42}$ or pTau. Again, only during the short-term memory task, an effect of decreasing $A \beta_{42}$ with increasing age became apparent when DMN FC was included in the model; there was no correlation between age and $A \beta_{42}$ otherwise.

\section{Neural Basis of Task-Driven Effective Connectivity and Resting-State Functional Connectivity}

Our results revealed different associations between AD biomarkers and FC patterns during task performance and resting states. Previous resting-state DMN connectivity studies have reported differences in healthy older adults (Jones et al., 2011; Ferreira and Busatto, 2013), patients with AD (Vemuri et al., 2011), and patients recovering from stroke (Grefkes and Ward, 2014), suggesting that DMN connectivity is altered in specific ways in aging and certain disease states. However, the physiological nature of FC during resting state is still controversial. For instance, it is not clear whether resting-state connectivity results from anatomical connections or synchronized oscillations in brain activity (Deco et al., 2011; Eickhoff and Grefkes, 2011). Meanwhile, task-driven cortical effective connectivity may be better understood in terms of physiological basis at the synaptic level (Rehme et al., 2013). 


\section{DMN \& Global Cognition (UDS T Scores)}
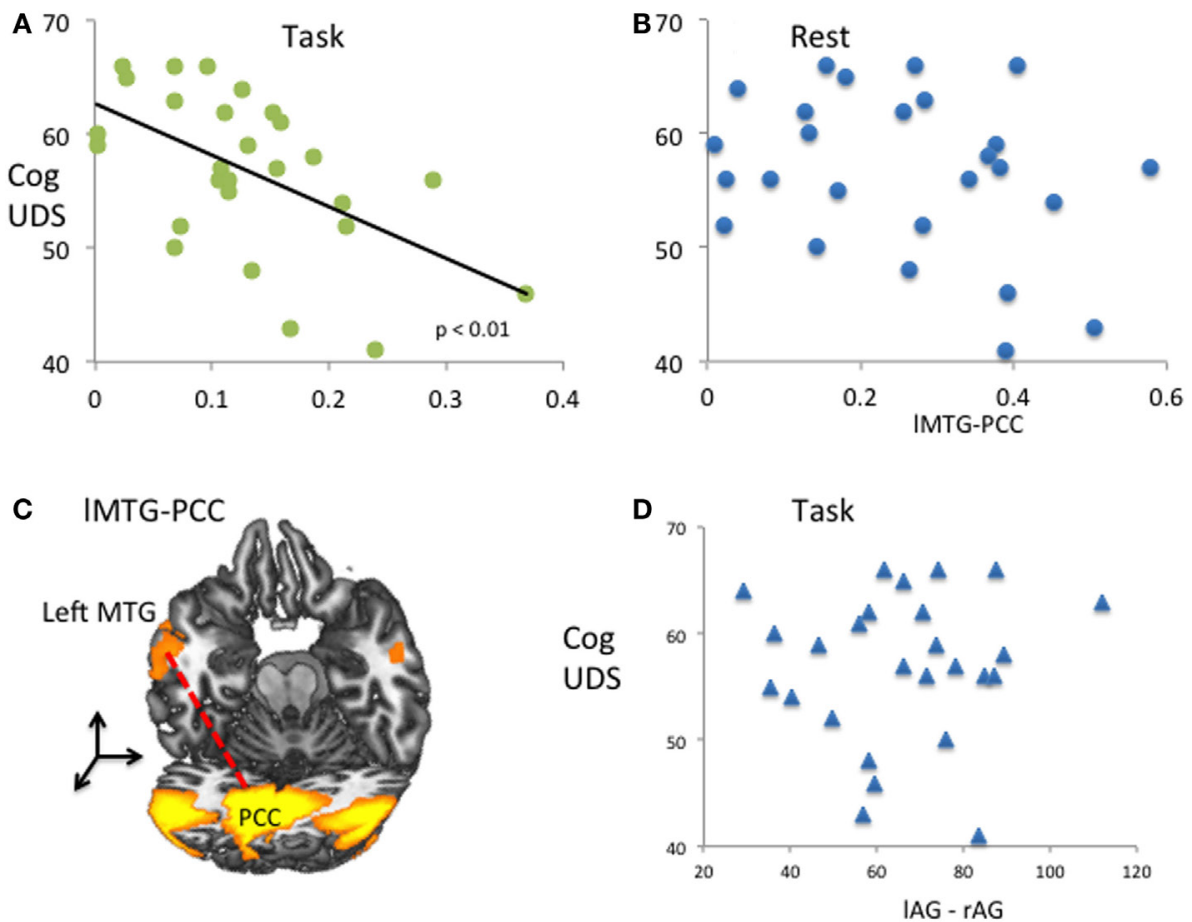

FIGURE 4 | DMN and global cognition (UDS $T$-Scores). (A) Significant correlation $(p<0.01$ ) was found during task between global cognitive scores and temporal-parietal (IMT-PCC) connectivity. (B) During resting state, no significant correlation was found between global cognitive scores and temporal-parietal connectivity. (C). Group averaged fMRI activity at the left MT and PCC/precuneus. (D). Bilateral IAG, correlated with pTau, was not correlated with global cognition.

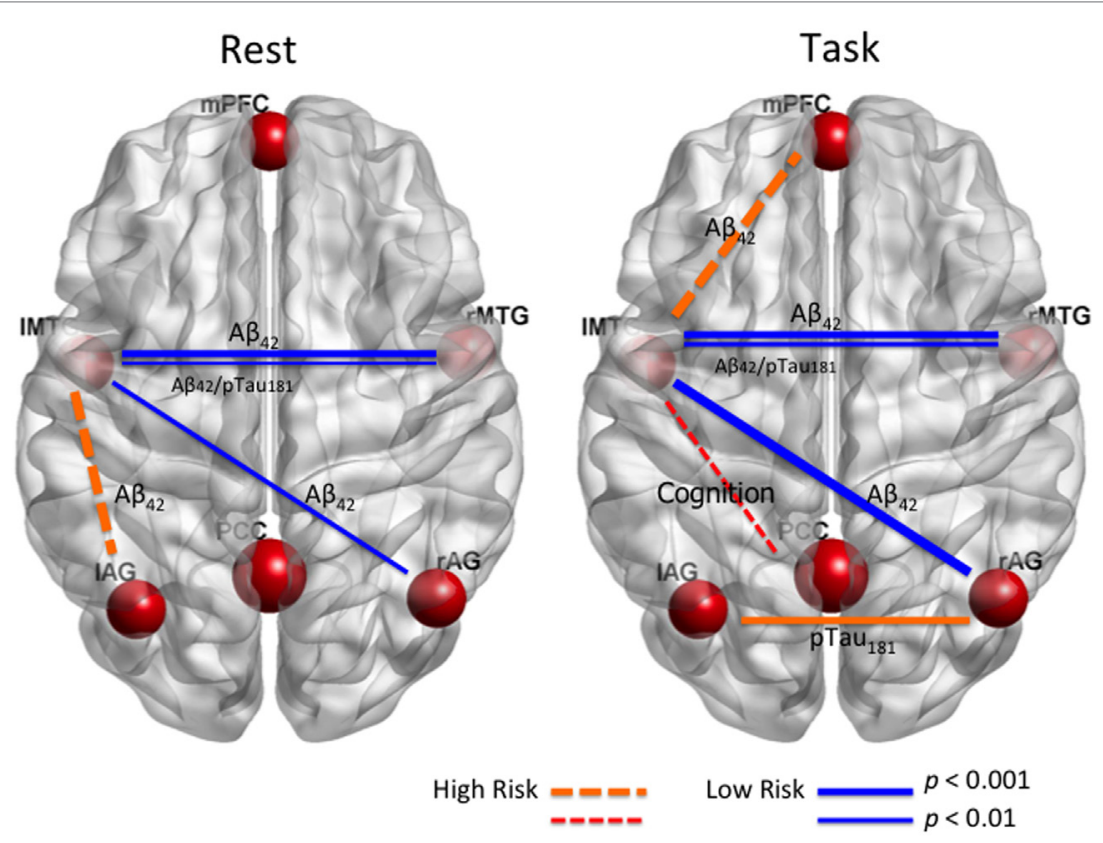

FIGURE 5 | Summary results of relations between functional brain connectivity within DMN during resting state and task, CSF biomarkers (A $\beta_{42}$ and pTau $\left.{ }_{181}\right)$, and global cognition ( $T$-scores) in cognitively normal older adults. Blue lines indicate low AD risk, and orange/red lines indicate high AD risk. The stronger the line, the more significant the connectivity with CSF biomarker or cognition. Solid line shows that the correlation was significant in more than one analysis. Left MTG appears to be a hub for connectivity sensitive to AD risk. 
Correlated brain activity is determined by ongoing and constantly shifting brain operations and processes engaging some networks and disengaging others (Fox et al., 2005). The DMN network activity is generally decreased during concentrated task performance and increased during the resting state, requiring the use of a relevant cognitive task as a modulator of DMN connectivity (Buckner et al., 2005, Buckner and Vincent, 2007). Since $\mathrm{A} \beta$ may preferentially affect component regions of the DMN and pTau is associated with synaptic degeneration, alterations in DMN connectivity may reflect multiple forms of subclinical AD pathology in both the resting and task states. Such alterations may be direct effects of the DMN itself or indirect effects mediated through synaptic interactions between DMN and other networks.

\section{Comparison of the Two Correlational Analyses in Relations to CSF Biomarkers}

We applied two measurements, zero-lag CC and TI, to assess FC. Interestingly, the current results indicate that TI is more strongly associated with CSF A $\beta_{42}$, while CC is more sensitive to levels of CSF $\mathrm{pTau}_{181}$ and global cognitive scores. Most of the previous analyses in the literature have applied the CC methods. The rationale for applying TI is that $\mathrm{FMRI}$ BOLD signals are time series. CC is not a time series-based measure but only exploits contemporaneous (zero lag) covariance structure of BOLD signal. The hallmark of time series is that current activity can affect the activity occurring at later times. Time series-based FC measurement, TI, takes into account the temporal dependence beyond zero lag (Wen et al., 2012). In general, the TI can capture the temporal relationship between BOLD time series, which is ignored by CC.

For the DMN during testing, both TI and CC identified positive relationships between IMTG and rMTG or mPFC and rAG with CSF $A \beta_{42}$ levels. Compared to CC, the TI method is more sensitive to the relationship of CSF $A \beta_{42}$ levels and other parameters (Table 3). In addition, TI revealed that increased IMTG-rAG during the resting state is associated with higher CSF $A \beta_{42}$ level, which was further confirmed by analysis during task-driven state. In our sample of high functioning cognitively intact older adults, age alone correlated with CSF $\mathrm{A} \beta_{42}$ only under the task performance while using the TI method to assess the FC. This correlation result of less CSFA $\beta_{42}$ with age is consistent with known literature. Curiously, traditional CC method shows more significant correlation with levels of p $\operatorname{Tau}_{181}$ (Tables 2 vs $\mathbf{3}$ ) and global cognitive score for some connections than TI (e.g., between bilateral AG).

\section{Bilateral Temporal Connectivity and AD Biomarker CSF A $\beta_{42}$}

Our most robust results indicate that CSF $A \beta_{42}$ level and $A \beta_{42}$ / $\mathrm{pTau}_{181}$ ratio during rest or task procedures are positively correlated with bilateral middle temporal connectivity (MTG, BA 21), which was detected in all six separate analyses. Thus, it appears that increased signaling between left MTG and right temporal and parietal cortices is more possible in older adults harboring lower levels of pathology. The opposite is true between left MTG and frontal $\mathrm{mPFC}$ and PPC within the left hemisphere.

The anterior middle temporal cortices are critical for higherlevel perception (Nakamura and Kubota, 1996), learning and memory (Arnold et al., 1994; Delacour, 1977; Lambon Ralph et al., 2009; Poettrich et al., 2009), language (Pobric et al., 2009; Schmidt and Seger, 2009), and novelty detection (Nakamura et al., 2000; Holeckova et al., 2008; McDonald et al., 2010). Additionally, the left anterior temporal cortex is considered a hub for semantic processing for object names (Shulman et al., 1997; Tsapkini et al., 2011). Typically, bilateral damage to anterior temporal cortices is required to produce a semantic deficit for object naming. Our visual working memory task includes elements of visual perception, language-assisted encoding, learning, short-term retention, and retrieval. The current findings suggest that $A \beta$ may contribute deficits in general functions present in DMN during both restingstate and cognitive tasks.

As $\mathrm{AD}$ biomarkers, CSF $\mathrm{A} \beta_{42}$ has been validated to have high agreement with $\mathrm{A} \beta$ detected using positron emission tomography (PET; FDA approved F-18) imaging (Landau et al., 2013; Palmqvist et al., 2014), although there may be discrepancies in preclinical and early symptomatic AD (Blennow et al., 2015). The accuracy of both PET and CSF biomarkers in $\mathrm{AD}$ diagnosis is in the $\sim 85 \%$ range (Mattsson et al., 2014). It would be interesting to validate the present finding using PET-based $\mathrm{AD}$ biomarkers.

\section{Bilateral Parietal Connectivity during Task and AD Biomarker CSF pTau 181}

Our present finding that CSF pTau correlates specifically with cortical connectivity during task performance, but not during rest, supports the idea that the presence of pTau may be associated with alterations within specific networks independently of $A \beta$. These alterations may be due to connections between DMN and other networks known to be affected earliest in $\mathrm{AD}$, e.g., entorhinal cortex in anteromedial temporal lobe. Interestingly, pTau ${ }_{181}$ showed no association with resting-state DMN but positively correlated with bilateral parietal (lAG-rAG) connectivity during task, i.e., only in models that included DMN connectivity during an active memory task.

In relation to predicting $\mathrm{AD}$ risk in cognitively intact older adults, the current results of connectivity changes unique to task-driven state are consistent with converging evidence that cognitive impairment correlates best with the burden of neocortical neurofibrillary tangles (Nelson et al., 2012). In symptomatic $\mathrm{AD}$, tau pathology is a more direct predictor of cognitive progression than $A \beta$ (Jagust, 2013; Koch et al., 2014); together with parietal white matter lesions, tau has been shown to contribute to early development of AD (Maruyama et al., 2004; Hertze et al., 2013). Our cohort of cognitively normal older adults who later developed $\mathrm{AD}$ have shown synaptic loss in projections from inferior temporal lobe (Scheff et al., 2011) to PCC with progression of AD (Scheff et al., 2015). The present data support the idea that there are synergistic effects of increased tau pathology and increased level of bilateral parietal activity during cognitive tasks (Hertze et al., 2013).

\section{Functional Connectivity Markers and Global Cognitive Status}

By contrast to CSF AD biomarkers, which showed no evidence of associations with cognitive status in the current study, FC between left temporal and parietal gyri during the short-term memory task 
strongly associated with overall cognitive status. Our findings support the idea that resting-state connectivity may reflect automatic and implicit cognitive activity, such as mind-wandering (Buckner and Vincent, 2007; Biswal et al., 2010), but it is not necessarily specific to higher-level cognitive tasks such as memory encoding and recall typically measured in neuropsychological tests (Broster et al., 2013). Resting-state connectivity during resting state was reported to be associated with CSF A $\beta_{42}$ in a cohort of cognitively intact older adults (Wang et al., 2013) and in AD patients (Li et al., 2013). These results further demonstrate that FC could provide a non-invasively measured intermediate phenotype reflecting effects of the presence of $A \beta$ and tau pathologies. This phenotype could serve as a biomarker of future cognitive decline and, possibly, a biomarker preceding threshold-defined abnormal levels of $A \beta$ and tau in healthy normal older adults.

\section{CONCLUSION}

In summary, we report that increased bilateral anterior middle temporal connectivity strongly correlated with higher CSF A $\beta_{42}$ level (lower $\mathrm{AD}$ risk) during both resting and task states in cognitively intact older adults. Increased bilateral parietal connectivity, only during a cognitive task, is associated with higher CSF pTau ${ }_{181}$ (higher AD risk). FC between left anterior temporal and inferior parietal lobes during the task is associated with lower cognitive function, even though cognitive performance remained well within the normal range in all participants. These new findings suggest that brain connectivity measures during resting and task states reflect different aspects of inter-regional interactions of brain states and $\mathrm{AD}$ risk in cognitively normal older adults. Brain connectivity measures associated with resting and task states may, thus, serve as complementary non-invasive markers for $\mathrm{A} \beta$ and tau deposits and early signs of cognitive decline in healthy older adults.

\section{REFERENCES}

Arnold, S. E., Hyman, B. T., and Van Hoesen, G. W. (1994). Neuropathologic changes of the temporal pole in Alzheimer's disease and Pick's disease. Arch. Neurol. 51, 145-150. doi:10.1001/archneur.1994.00540140051014

Biswal, B., Yetkin, F. Z., Haughton, V. M., and Hyde, J. S. (1995). Functional connectivity in the motor cortex of resting human brain using echo-planar MRI. Magn. Reson. Med. 34, 537-541. doi:10.1002/mrm.1910340409

Biswal, B. B., Mennes, M., Zuo, X. N., Gohel, S., Kelly, C., Smith, S. M., et al. (2010). Toward discovery science of human brain function. Proc. Natl. Acad. Sci. U.S.A. 107, 4734-4739. doi:10.1073/pnas.0911855107

Blennow, K., Mattsson, N., Schöll, M., Hansson, O., and Zetterberg, H. (2015). Amyloid biomarkers in Alzheimer's disease. Trends Pharmacol. Sci. 36, 297-309. doi:10.1016/j.tips.2015.03.002

Broster, L. S., Li, J., Smith, C. D., Jicha, G. A., Schmitt, F. A., and Jiang, Y. (2013). Repeated retrieval during working memory is sensitive to amnestic mild cognitive impairment. J. Clin. Exp. Neuropsychol. 35, 946-959. doi:10.1080/1 3803395.2013.838942

Buckner, R. L., Snyder, A. Z., Shannon, B. J., LaRossa, G., Sachs, R., Fotenos, A. F., et al. (2005). Molecular, structural, and functional characterization of Alzheimer's disease: evidence for a relationship between default activity, amyloid, and memory. J. Neurosci. 25, 7709-7717. doi:10.1523/ JNEUROSCI.2177-05.2005

Buckner, R. L., and Vincent, J. L. (2007). Unrest at rest: default activity and spontaneous network correlations. Neuroimage 37, 1091-1096. doi:10.1016/j. neuroimage.2007.01.010

\section{AUTHOR CONTRIBUTIONS}

YJ designed the study, contributed to data analysis, interpretations, and wrote the paper. $\mathrm{HH}$ conducted neuroimaging data analysis and contributed to data interpretation and writeup. EA conducted overall statistical analysis and writeup. LB contributed to experimental design, data collection, and analysis. GAJ contributed to neuroimaging and CSF data collection and analysis. FAS and RK contributed to neuropsychological data collection, statistical analysis, and writeup. AA and DP contributed to imaging data collection, analysis, and writeup. LV, BG, and PN contributed to data collection, interpretation, and writeup. CS contributed to imaging and CSF data collection, interpretation, and writeup. MD contributed to development of new computational methods, data analysis, interpretations, and writeup.

\section{ACKNOWLEDGMENTS}

We thank the volunteers in the "BRAiNs" program at the University of Kentucky Sanders-Brown Center on Aging for their participation in the study, and M. Baker for editorial assistance.

\section{FUNDING}

The study was supported by funding from the National Institute of Health of the United States, National Institute of Aging P30 AG028383 and R21 AG044862.

\section{SUPPLEMENTARY MATERIAL}

The Supplementary Material for this article can be found online at http://journal.frontiersin.org//article/10.3389/ fnagi.2016.00015

Crary, J. F., Trojanowski, J. Q., Schneider, J. A., Abisambra, J. F., Abner, E. L., Alafuzoff, I., et al. (2014). Primary age-related tauopathy (PART): a common pathology associated with human aging. Acta Neuropathol. 128, 755-766. doi:10.1007/s00401-014-1349-0

Deco, G., Jirsa, V. K., and McIntosh, A. R. (2011). Emerging concepts for the dynamical organization of resting-state activity in the brain. Nat. Rev. Neurosci. 12, 43-56. doi:10.1038/nrn2961

Delacour, J. (1977). Role of temporal lobe structures in visual short-term memory, using a new test. Neuropsychologia 15, 4-5. doi:10.1016/0028-3932(77)90072-0

Dickerson, B. C., and Wolk, D. A. (2012). MRI cortical thickness biomarker predicts AD-like CSF and cognitive decline in normal adults. Neurology 78, 84-90. doi:10.1212/WNL.0b013e31823efc6c

Ding, M., Chen, Y., and Bressler, S. L. (2006). "Ch 17: Granger causality: basic theory and application to neuroscience," in Handbook of Time Series Analysis, eds Schelter B., Winderhalder M., and Timmer J. (Weinheim: Wiley-VCH Verlag), 17.

Eickhoff, S. B., and Grefkes, C. (2011). Approaches for the integrated analysis of structure, function and connectivity of the human brain. Clin. EEG Neurosci. 42, 107-121. doi:10.1177/155005941104200211

Ferreira, L. K., and Busatto, G. F. (2013). Resting-state functional connectivity in normal brain aging. Neurosci. Biobehav. Rev. 37, 384-400. doi:10.1016/j. neubiorev.2013.01.017

Fox, M. D., Snyder, A. Z., Vincent, J. L., Corbetta, M., Van Essen, D. C., and Raichle, M. E. (2005). The human brain is intrinsically organized into dynamic, anticorrelated functional networks. Proc. Natl. Acad. Sci. U.S.A. 102, 9673-9678. doi:10.1073/pnas.0504136102 
Gold, B. T., Zhu, Z., Brown, C. A., Andersen, A. H., LaDu, M. J., Tai, L., et al. (2014). White matter integrity is associated with CSF markers of AD in normal adults. Neurobiol. Aging 35, 2263-2271. doi:10.1016/j.neurobiolaging.2014.04.030

Grady, C. L., Furey, M. L., Pietrini, P., Horwitz, B., Schapiro, M. B., and Rapoport, S. I. (2001). Altered functional connectivity among prefrontal and medial temporal regions and impaired short-term memory in Alzheimer's disease. Brain 124, 739-756. doi:10.1093/brain/124.4.739

Grefkes, C., and Ward, N. S. (2014). Cortical reorganization after stroke: how much and how functional? Neuroscientist. 20, 56-70. doi:10.1177/1073858413491147

Greicius, M. D., Srivastava, G., Reiss, A. L., and Menon, V. (2004). Default-mode network activity distinguishes Alzheimer's disease from healthy aging: evidence from functional MRI. Proc. Natl. Acad. Sci. U.S.A. 101, 4637-4642. doi:10.1073/ pnas.0308627101

Hertze, J., Palmqvist, S., Minthon, L., and Hansson, O. (2013). Tau pathology and parietal white matter lesions have independent but synergistic effects on early development of Alzheimer's disease. Dement. Geriatr. Cogn. Dis. Extra. 3, 113-122. doi:10.1159/000348353

Holeckova, I., Fischer, C., Morlet, D., Delpuech, C., Costes, N., and Mauguiere, F. (2008). Subject's own name as a novel in a MMN design: a combined ERP and PET study. Brain Res. 1189, 152-165. doi:10.1016/j. brainres.2007.10.091

Hu, Y., Chen, X., Gu, H., and Yang, Y. (2012). Resting-state glutamate and GABA concentrations predict task-induced deactivation in the default mode network. J. Neurosci. 33, 18566-18573. doi:10.1523/JNEUROSCI.1973-13.2013

Jack, C. R. Jr., Knopman, D. S., Jagust, W. J., Petersen, R. C., Weiner, M. W., Aisen, P. S., et al. (2013). Tracking pathophysiological processes in Alzheimer's disease: an updated hypothetical model of dynamic biomarkers. Lancet Neurol. 12, 207-216. doi:10.1016/S1474-4422(12)70291-0

Jagust, W. (2013). Vulnerable neural systems and the borderland of brain aging and neurodegeneration. Neuron 77, 219-234. doi:10.1016/j.neuron.2013.01.002

Jiang, Y., Haxby, J. V., Martin, A., Ungerleider, L. G., and Parasuraman, R. (2000). Complementary neural mechanisms for tracking items in human working memory. Science 287, 643-646. doi:10.1126/science.287.5453.643

Jones, D. T., Machulda, M. M., Vemuri, P., McDade, E. M., Zeng, G., Senjem, M. L., et al. (2011). Age-related changes in the default mode network are more advanced in Alzheimer disease. Neurology 77, 1524-1531. doi:10.1212/ WNL.0b013e318233b33d

Kandimalla, R. J., Prabhakar, S., Binukumar, B. K., Wani, W. Y., Gupta, N., and Sharma, D. R. (2011). Apo-Eع4 allele in conjunction with A $\beta 42$ and tau in CSF: biomarker for Alzheimer's disease. Curr. Alzheimer Res. 8, 187-196. doi:10.2174/156720511795256071

Kandimalla, R. J. L., Prabhakar, S., Wani, W. Y., Kaushal, A., Gupta, N., Sharma, D. R., et al. (2013). CSF p-Tau levels in the prediction of Alzheimer's disease. Biol. Open 2, 1119-1124. doi:10.1242/bio.20135447

Koch, K., Myers, N. E., Gottler, J., Pasquini, L., Grimmer, T., Forster, S., et al. (2014). Disrupted intrinsic networks link amyloid- $\beta$ pathology and impaired cognition in prodromal Alzheimer's disease. Cereb. Cortex 25, 4678-4688. doi:10.1093/ cercor/bhu151

Kovacs, G. G., Ferrer, I., Grinberg, L. T., Alafuzoff, I., Attems, J., Budka, H., et al. (2016). Aging-related tau astrogliopathy (ARTAG): harmonized evaluation strategy. Acta Neuropathol. 131, 87-102. doi:10.1007/s00401-015-1509-x

Lambon Ralph, M. A., Pobric, G., and Jefferies, E. (2009). Conceptual knowledge is underpinned by the temporal pole bilaterally: convergent evidence from rTMS. Cereb. Cortex 19, 832-838. doi:10.1093/cercor/bhn131

Landau, S. M., Lu, M., Joshi, A. D., Pontecorvo, M., Mintun, M. A., Trojanowski, J. Q., et al. (2013). Comparing PET imaging and CSF measurements of A $\beta$. Ann. Neurol. 74, 826-836. doi:10.1002/ana.23908

Li, X., Li, T. Q., Andreasen, N., Wiberg, M. K., Westman, E., and Wahlund, L. O. (2013). Ratio of $A \beta 42 / P-t_{10 u} 18$ in CSF is associated with aberrant default mode network in AD. Sci Rep 3, 1339. doi:10.1038/srep01339

Maruyama, M., Matsui, T., Tanji, H., Nemoto, M., Tomita, N., Ootsuki, M., et al. (2004). Cerebrospinal fluid tau protein and periventricular white matter lesions in patients with mild cognitive impairment: implications for 2 major pathways. Arch. Neurol. 61, 716-720. doi:10.1001/archneur.61.5.716

Mathews, M., Abner, E., Kryscio, R., Jicha, G., Cooper, G., Smith, C., et al. (2014). Diagnostic accuracy and practice effects in the National Alzheimer's Coordinating Center uniform data set neuropsychological battery. Alzheimers Dement. 10, 675-683. doi:10.1016/j.jalz.2013.11.007
Mattsson, N., Insel, P. S., Landau, S., Jagust, W., Donohue, M., Shaw, L. M., et al. (2014). Diagnostic accuracy of CSF Ab42 and florbetapir PET for Alzheimer's disease. Ann. Clin. Transl. Neurol. 1, 534-543. doi:10.1002/acn3.81

McDonald, C. R., Thesen, T., Carlson, C., Blumberg, M., Girard, H. M., Trongnetrpunya, A., et al. (2010). Multimodal imaging of repetition priming: using $\mathrm{fMRI}, \mathrm{MEG}$, and intracranial EEG to reveal spatiotemporal profiles of word processing. Neuroimage 53, 707-717. doi:10.1016/j. neuroimage.2010.06.069

Morris, J., Weintraub, S., Chui, H. C., Cummings, J., DeCaril, C., Ferris, S., et al. (2006). The uniform data set (UDS): clinical and cognitive variables and descriptive data from Alzheimer Disease Centers. Alzheimer Dis. Assoc. Disord. 20, 210-216. doi:10.1097/01.wad.0000213865.09806.92

Nakamura, K., Kawashima, R., Sato, N., Nakamura, A., Sugiura, M., Kato, T., et al. (2000). Functional delineation of the human occipito-temporal areas related to face and scene processing. A PET study. Brain 123, 1903-1912. doi:10.1093/ brain/123.9.1903

Nakamura, K., and Kubota, K. (1996). The primate temporal pole: its putative role in object recognition and memory. Behav. Brain Res. 77, 53-77. doi:10.1016/0166-4328(95)00227-8

Nelson, P. T., Alafuzoff, I., Bigio, E. H., Bouras, C., Braak, H., Cairns, N. J., et al. (2012). Correlation of Alzheimer's disease neuropathologic changes with cognitive status: a review of the literature. J. Neuropathol. Exp. Neurol. 71, 362-381. doi:10.1097/NEN.0b013e31825018f7

Palmqvist, S., Zetterberg, H., Blennow, K., Vestberg, S., Andreasson, U., Brooks, D. J., et al. (2014). Accuracy of brain amyloid detection in clinical practice using cerebrospinal fluid beta-amyloid 42: a cross validation study against amyloid positron emission tomography. JAMA Neurol. 71, 1282-1289. doi:10.1001/ jamaneurol.2014.1358

Palop, J. J., and Mucke, L. (2010). Amyloid-beta-induced neuronal dysfunction in Alzheimer's disease: from synapses toward neural networks. Nat. Neurosci. 13, 812-818. doi:10.1038/nn.2583

Parasuraman, R., and Jiang, Y. (2012). Individual differences in cognition, affect, and performance: behavioral, neuroimaging, and molecular genetic approaches. Neuroimage 59, 70-82. doi:10.1016/j.neuroimage.2011.04.040

Pobric, G., Lambon Ralph, M. A., and Jefferies, E. (2009). The role of the anterior temporal lobes in the comprehension of concrete and abstract words: rTMS evidence. Cortex 45, 1104-1110. doi:10.1016/j.cortex.2009.02.006

Poettrich, K., Weiss, P. H., Werner, A., Lux, S., Donix, M., Gerber, J., et al. (2009). Altered neural network supporting declarative long-term memory in mild cognitive impairment. Neurobiol. Aging 30, 284-298. doi:10.1016/j. neurobiolaging.2007.05.027

Quigley, H., Colloby, S. J., and O'Brien, J. T. (2011). PET imaging of brain amyloid in dementia: a review. Int. J. Geriatr. Psychiatry 26, 991-999. doi:10.1002/gps.2640

Raichle, M. E., MacLeod, A. M., Snyder, A. Z., Powers, W. J., Gusnard, D. A., and Shulman, G. L. (2001). A default mode of brain function. Proc. Natl. Acad. Sci. U.S.A. 98, 676-682. doi:10.1073/pnas.98.2.676

Rehme, A. K., Eickhoff, S. B., and Grefkes, C. (2013). State-dependent differences between functional and effective connectivity of the human cortical motor system. Neuroimage 67, 237-246. doi:10.1016/j.neuroimage.2012.11.027

Scheff, S. W., Price, D. A., Ansari, M. A., Roberts, K. N., Schmitt, F. A., Ikonomovic, M. D., et al. (2015). Synaptic change in the posterior cingulate gyrus in the progression of Alzheimer's disease. J. Alzheimers Dis. 43, 1073-1090. doi:10.3233/ JAD-141518

Scheff, S. W., Price, D. A., Schmitt, F. A., Scheff, M. A., and Mufson, E. J. (2011). Synaptic loss in the inferior temporal gyrus in mild cognitive impairment and Alzheimer's disease. J. Alzheimers Dis. 24, 547-557. doi:10.3233/ JAD-2011-101782

Schmidt, G. L., and Seger, C. A. (2009). Neural correlates of metaphor processing: the roles of figurativeness, familiarity and difficulty. Brain Cogn. 71, 375-386. doi:10.1016/j.bandc.2009.06.001

Schmitt, F. A., Nelson, P. T., Abner, E., Scheff, S., Jicha, G. A., Smith, C., et al. (2012). University of Kentucky Sanders-Brown healthy brain aging volunteers: donor characteristics, procedures and neuropathology. Curr. Alzheimer Res. 9, 724-733. doi:10.2174/156720512801322591

Shulman, G. L., Fiez, J. A., Corbetta, M., Buckner, R. L., Miezin, F. M., Raichle, M. E., et al. (1997). Common blood flow changes across visual tasks: II. Decreases in cerebral cortex. J. Cogn. Neurosci. 9, 648-663. doi:10.1162/ jocn.1997.9.5.648 
Sjögren, M., Vanderstichele, H., Agren, H., Zachrisson, O., Edsbagge, M., Wikkelso, C., et al. (2001). Tau and Abeta42 in cerebrospinal fluid from healthy adults 21-93 years of age: establishment of reference values. Clin. Chem. 47, 1776-1781.

Snodgrass, J. G., and Vanderwart, M. (1980). A standardized set of 260 pictures: norms for name agreement, image agreement, familiarity, and visual complexity. J. Exp. Psychol. Hum. Learn. 6, 174-215. doi:10.1037/0278-7393.6.2.174

Sperling, R. A., LaViolette, P. S., O’Keefe, K., O’Brien, J., Rentz, D. M., Pihlajamaki, M., et al. (2009). Amyloid deposition is associated with impaired default network function in older persons without dementia. Neuron 63, 178-188. doi:10.1016/j.neuron.2009.07.003

Tai, L. M., Bilousova, T., Jungbauer, L., Roeske, S. K., Youmans, K. L., Yu, C., et al. (2013). Levels of soluble apolipoprotein E/amyloid- $\beta$ (A $\beta$ ) complex are reduced and oligomeric A $\beta$ increased with APOE4 and Alzheimer disease in a transgenic mouse model and human samples. J. Biol. Chem. 288, 5914-5926. doi:10.1074/jbc.M112.442103

Tibshirani, R. (1996). Regression shrinkage and selection via the lasso. J. R. Stat. Soc. 58, 267.

Tsapkini, K., Frangakis, C. E., and Hillis, A. E. (2011). The function of the left anterior temporal pole: evidence from acute stroke and infarct volume. Brain 134, 3094-3105. doi:10.1093/brain/awr050

Vemuri, P., Jones, D. T., and Jack, C. R. (2011). Resting state functional MRI in Alzheimer's disease. Alzheimers Res. Ther. 4, 2. doi:10.1186/alzrt100

Wang, L., Brier, M. R., and Snyder, A. Z. (2013). Cerebrospinal fluid A $\beta 42$, phosphorylated tau181, and resting-state functional connectivity. JAMA Neurol. 70, 1242-1248. doi:10.1001/jamaneurol.2013.3253
Weintraub, S., Salmon, D. P., Mercaldo, N., Ferris, S., Graff-Radford, N., Chui, H., et al. (2009). The Alzheimer's Disease Centers' Uniform Data Set (UDS): the neuropsychological test battery. Alzheimer's Dis Assoc Disord. 23, 91-101.

Wen, X., Mo, J., and Ding, M. (2012). Exploring resting-state functional connectivity with total interdependence. Neuroimage 60, 1587-1595. doi:10.1016/j. neuroimage.2012.01.079

Wirth, M., Madison, C. M., Rabinovici, G. D., Landau, S. M., and Jagust, W. J. (2013). Alzheimer's disease neurodegenerative biomarkers are associated with decreased cognitive function but not $\beta$-amyloid in cognitively normal older individuals. J. Neurosci. 33, 5553-5563. doi:10.1523/JNEUROSCI.4409-12.2013

Conflict of Interest Statement: The authors declare that the research was conducted in the absence of any commercial or financial relationships that could be construed as a potential conflict of interest.

The Reviewer Subodh Kumar declares that, despite being affiliated with the same institution as the handling Editor P. Hemachandra Reddy, the review process was handled objectively.

Copyright (C) 2016 Jiang, Huang, Abner, Broster, Jicha, Schmitt, Kryscio, Andersen, Powell, Van Eldik, Gold, Nelson, Smith and Ding. This is an open-access article distributed under the terms of the Creative Commons Attribution License (CC BY). The use, distribution or reproduction in other forums is permitted, provided the original author(s) or licensor are credited and that the original publication in this journal is cited, in accordance with accepted academic practice. No use, distribution or reproduction is permitted which does not comply with these terms. 